\title{
Role of negative experiences in past relationships and adverse childhood experiences in morbid jealousy
}

\author{
S Karunarathne, N Liyanage, A Rodrigo
}

\section{Abstract \\ Background}

Morbid jealousy is a relatively common and disabling mental health entity. Psychological theories suggest experiences of being cheated-on in previous relationships and childhood experience of parents having or being accused of having extramarital relationships as aetiological factors of morbid jealousy. However this has not been studied adequately.

\section{Method}

A retrospective case control study was conducted based on clinical records of patients admitted to the psychiatric unit in a tertiary care hospital in Sri Lanka over a 14 month period. Patients with morbid jealousy were considered as cases and those admitted with other psychiatric diagnoses were considered as controls.

Results

Of 2708 patients, 131 (4.8\%) were identified to have morbid jealousy. In patients with morbid jealousy, a majority were males, average age was 42.8 years and schizophrenia was the commonest diagnosis. There was a statistical significant association between morbid jealousy and past experiences of being cheated-on or childhood experiences of parents having or being accused of having extra-marital relationships.

\section{Discussion}

Morbid jealousy was a common presentation. The significant association between morbid jealousy and above experiences could be due to a true association, recall or interpretation bias by patients with morbid jealousy, or more diligent exploration by clinicians when it comes to patient with morbid jealousy. Except for the last explanation, this association has treatment implications.

Keywords: morbid jealousy, aetiology, previous relationships, childhood experience

SL J Psychiatry 2017; 8(1): 20-23

\section{Background}

Morbid jealousy describes a range of irrational thoughts and emotions, together with associated unacceptable or extreme behaviour, in which the dominant theme is a preoccupation with a partner's sexual unfaithfulness based on unfounded evidence (1). This preoccupation can be a delusion, obsession or an overvalued idea (2).

Although morbid jealousy has been considered a rare diagnosis, mental health clinicians encounter it frequently (2). While the worldwide prevalence of morbid jealousy is unknown, Kapugama et al., (2013) reported morbid jealousy to be a common finding (17.1\%) among inpatients of the University Psychiatry Unit of National Hospital of Sri Lanka (3). A study conducted in Colombo, Sri Lanka reported that the local clinical presentation of morbid jealousy was largely similar to presentations described in the international, largely Western, literature (4).

Morbid jealousy is a condition associated with significant negative consequences. Confirmatory behaviours are common and focus on the investigation of the partner's fidelity. These behaviours include searching the partner's clothes, mobile phones and other personal belongings to find evidence of infidelity, surprise visits to partner's workplace and stalking. In extreme circumstances the person can become violent to extract a confession from the partner. Repeated denials may provoke extreme anger and violence. The constantly suffering partner can even make a false confession leading to more violence. The partner may develop mental disorders such as anxiety and depression. Suicide and self-harm are not uncommon among people suffering from morbid jealousy. Children of a morbidly jealous parent may be subjected to physical and emotional abuse (2).

There are many proposed aetiological and predisposing factors for morbid jealousy. It is often comorbid with other psychiatric disorders, with alcohol use disorders being commonly associated. With regard to theories of development, psychodynamic theories suggest that the actual, supposed or threatened loss of affection by parents will introduce the child to his or her first experience of jealousy, which may make them more vulnerable to development of jealousy later on their lives (5). Docherty and Ellis, in a case study, described three 
morbidly jealous men who saw their mothers engage in extramarital sexual activity during their adolescence (6). Mathes stated in his cognitive model of jealousy, that previous negative experience in a relationship may play a significant role in formation of jealousy through feelings of insecurity or inadequacy and low-esteem (7).

Possible associations between experiences about being cheated-on in previous relationships, or childhood experiences of extramarital relationships in parents, and later development of morbid jealousy has rarely been studied.

\section{Method}

This was a retrospective case control study, based on case notes of all the patients admitted to the Psychiatry Unit of Colombo North Teaching Hospital over a 14month period, between January 2014 and February 2015. Of the 3315 admissions during this period, 2797 records were included in the study, since patient records were not available for the remainder. A further 89 records were excluded as these were re-admissions within one week of discharge. Patient records were perused manually by a consultant psychiatrist and registrar in psychiatry.

Cases were defined as patients with symptoms of morbid jealousy as defined by Cobb, irrespective of their other diagnoses and irrespective of whether morbid jealousy was the main presenting problem or not (1). All remaining patient records, which were not readmissions, were considered as controls. Patient records of both cases and controls were perused for past experiences of being cheated-on by previous partners and childhood experience of parents having or being accused of having extramarital relationships. The psychiatric diagnoses of those with morbid jealousy were recorded.

\section{Results}

Of the 2708 patients records $(71.9 \%$, males $n=1948)$ included in the study, 131 (4.8\%) were identified to have symptoms of morbid jealousy. A majority of those with morbid jealousy were males $(80.0 \%, n=105)$ and the average age was 42.8 years (ranging between 23-76 years). Schizophrenia was the most common diagnosis amongst those with morbid jealousy (Table 1).

\begin{tabular}{|lrr|}
\hline Table 1. Psychiatric diagnosis of individuals with morbid jealousy & \\
\hline Diagnosis & $\mathrm{n}$ & $\%$ \\
\hline Monosymptomatic delusional disorder & 4 & 3.0 \\
Schizophrenia or schizoaffective disorder & 41 & 31.3 \\
Depressive disorder & 24 & 18.3 \\
OCD & 3 & 2.3 \\
Mental and behavioural disorder due to substance use & 18 & 3.8 \\
Dementia & 5 & 27.5 \\
Diagnosis not clear & 36 & 100 \\
\end{tabular}

\begin{tabular}{|c|c|c|c|}
\hline $\begin{array}{l}\text { Experience of being cheated on in } \\
\text { previous relationships }\end{array}$ & $\begin{array}{l}\text { Cases } \\
(n=131)\end{array}$ & $\begin{array}{l}\text { Controls } \\
(n=2577)\end{array}$ & \\
\hline Yes & 34 (26\%) & $256(10 \%)$ & $\chi^{2}=33.46$ \\
\hline No & 97 (74\%) & 2321 (90\%) & $p<0.01$ \\
\hline $\begin{array}{l}\text { Childhood experience of parents having or being } \\
\text { accused of having extra-marital relationship }\end{array}$ & $\begin{array}{l}\text { Cases } \\
(n=131)\end{array}$ & $\begin{array}{l}\text { Controls } \\
(n=2577)\end{array}$ & \\
\hline Yes & 41 (31\%) & 211 (8\%) & $\chi^{2}=78.89$ \\
\hline No & 90 (69\%) & 2366 (92\%) & $p<0.01$ \\
\hline
\end{tabular}

Cases - patients with morbid jealousy; Controls - patients without morbid jealousy; $\chi^{2}=$ chi square statistic 
Previous experiences of being cheated-on were recorded in $25.9 \%(n=34 / 131)$ of patients with morbid jealousy and in $9.9 \% \quad(n=256 / 2577)$ of controls. Similarly childhood experience of parents having or being accused of having extra-marital relationships was recorded in $35.9 \%(n=47 / 131)$ of cases and 15.5\% $(n=399 / 2577)$ of controls. There was a statistical significant association between morbid jealousy and past experiences of being cheated on $(p<0.01)$, as well as childhood experiences of parents having or being accused of having extra-marital relationships $(p<0.01)$ (Table 2).

\section{Discussion}

The findings of this study suggest that morbid jealousy is a common finding among psychiatric inpatients. Kapugama et al., too reported a high prevalence of morbid jealousy (17.1\%) in a smaller cohort of Sri Lankan patients (3). This was far greater than the prevalence of delusional jealousy $(0.5 \%$ and $1.1 \%)$ in patients admitted to the Psychiatric Hospital, University of Munich, Germany during two different periods $(8,9)$. While the more inclusive nature of definition of morbid jealousy used in Sri Lankan studies may account for the difference, the role played by Sri Lankan culture cannot be excluded (10). In this study, a majority of patients with morbid jealousy were males, even after accounting for a higher number male admissions during the study period. In previous studies there is no clear consensus on gender predilection of morbid jealousy $(2,3,8,9)$ Our findings regarding the diagnosis of individuals with morbid jealousy was largely similar to previous studies, with the most frequent diagnosis being schizophrenia followed by mood disorders $(3,8,9)$. It is interesting to note that in 36 patients a clear diagnosis was not documented, highlighting the deficiencies of record keeping.

Past experiences of being cheated-on or childhood experience of parents having or being accused of having extra-marital relationships was recorded more frequently in the case notes patients with morbid jealousy when compared to those without. One possible explanation is that these factors being causal for morbid jealousy, as suggested by Mathes and Docherty and Ellis $(6,7)$. On the other hand, patients with morbid jealousy may recall such occurrences more readily, may have distorted or delusional memory about such events; or clinicians may have explored these aspects in greater depth in patients with morbid jealousy.

The role of cognitive biases in the development, maintenance, relapse and recurrence of mental illnesses, particularly in depression, has been established (11). Individuals with mental illnesses have attention, interpretation, and memory biases for relevant negative information (12). These individuals have greater propensity to interpret information in a negative manner and a tendency to recall negative information when compared with healthy individuals $(13,14)$. These cognitive biases and memories are not mere symptoms or mood-dependent correlates of the disorder, but causal antecedents and perpetuating factors, hence important in treatment (12).

\section{Limitations}

There are several limitations in our study in addition to the usual shortcomings of retrospective analysis. Although clinical records are generally considered to be comprehensive and reliable, some records may have limited validity. All patients with morbid jealousy may not be recorded as such, particularly those presenting with other symptoms. As mentioned previously the two features of previous experience of being cheated on and childhood experience of parents having or being accused of having extra-marital relationships may have been explored more by clinicians, when interviewing patients presenting with features of morbid jealousy, and this may have influenced our findings.

\section{Conclusions}

Our study suggests, clinician exploration bias notwithstanding, individuals with morbid jealousy have either experienced or are more likely to recall incidents of past experiences of being cheated-on or childhood experience of parents having or being accused of having extra-marital relationships. This area has rarely been studied before. In light of this new knowledge, we believe such clinical information should be carefully explored and used in treatment, including cognitive restructuring.

\section{Disclosure statement}

None declared

S Karunarathna and A Rodrigo, Department of Psychiatry, Faculty of Medicine, University of Kelaniya, Ragama, Sri Lanka

N Liyanage, University Psychiatry Unit, National Hospital, Colombo, Sri Lanka

Corresponding author: A Rodrigo

Email: asirir2000@yahoo.com

http://orcid.org/0000-0003-3962-0592

\section{References}

1. Cobb J. Morbid jealousy. Br J Hosp Med. 1979; 21(5): 511-8.

2. Kingham M, Gordon H. Aspects of morbid jealousy. Adv Psychiatric Treatment 2004; 10: 107-15.

3. Kapugama C, Chathurie Suraweera C, Kotalawala W, Wijesiri V, Dalpatadu M, Hanwella R. Prevelence of morbid 
Role of negative experiences in past relationships and adverse childhood experiences in morbid jealousy

jealousy among inpatients in a psychiatry unit in Sri Lanka. SL J Psychiatry 2013; 4 (2): 38-40

4. De Silva D, De Silva P. Morbid jealousy in an Asian country: a clinical exploration from Sri Lanka Int Rev Psychiatry 1999; 11 (2-3), 116-121.

5. Clanton G, Kosins DJ. Developmental correlates of jealousy. in: Salvoey P, Ed. The Psychology of Jealousy and Envy. New York: Guilford, 1991; 132-147.

6. Docherty JP, Ellis J. A new concept and finding in morbid jealousy. Am J Psychiatry 1979; 133: 679-83.

7. Mathes EW. (1991) A cognitive theory of jealousy. in: Salvoey P, Ed. The Psychology of Jealousy and Envy. New York: Guilford, 1991; 271-86.

8. Soyka M, Schmidt P. Prevalence of delusional jealousy in psychiatric disorders. J Forensic Sci 2011; 56(2): 450-2.

9. Soyka M, Naber G, Völcker A. Prevalence of delusional jealousy in different psychiatric disorders. An analysis of 93 cases. Br J Psychiatry 1991; 158: 549-53.

10. Bhugra D. Cross-cultural aspects of jealousy. Int Rev Psychiatry 1993; 5: 271-80.

11. Gotlib IH, Joormann J. Cognition and depression: current status and future directions. Annu Rev Clin Psychol 2010; 6: 285-312.

12. Everaert, J., Koster, E.H., Derakshan, N. (2012). The combined cognitive bias hypothesis in depression. Clin Psychol Rev 2012; 32(5): 413-24.

13. Colombel F. Memory bias and depression: a critical commentary. Encephale 2007; 33(3 Pt 1): 242-8.

14. De Raedt R., Koster EH. Understanding vulnerability for depression from a cognitive neuroscience perspective: A reappraisal of attentional factors and a new conceptual framework. Cogn Affect Behav Neurosci 2010; 10(1): 50-70. 